\title{
Application of a joint and iterative MMSE-based estimation of SNR and frequency-selective channel for OFDM systems
}

\author{
Vincent Savaux ${ }^{1} 2^{*}$, Yves Louët ${ }^{2}$, Moïse Djoko-Kouam ${ }^{1}$ and Alexandre Skrzypczak ${ }^{3}$
}

\begin{abstract}
This article presents an iterative minimum mean square error- (MMSE-) based method for the joint estimation of signal-to-noise ratio (SNR) and frequency-selective channel in an orthogonal frequency division multiplexing (OFDM) context. We estimate the SNR thanks to the MMSE criterion and the channel frequency response by means of the linear MMSE (LMMSE). As each estimation requires the other one to be performed, the proposed algorithm is iterative. In this article, a realistic case is considered; i.e., the channel covariance matrix used in LMMSE is supposed to be totally unknown at the receiver and must be estimated. We will theoretically prove that the algorithm converges for a relevantly chosen initialization value. Furthermore simulations show that the algorithm quickly converges to a solution that is close to the one in which the covariance matrix is perfectly known. Compared to existing SNR estimation methods, the algorithm improves the trade-off between the number of required pilots and the SNR estimation quality.
\end{abstract}

Keywords: OFDM; Estimation; Signal-to-noise ratio; Frequency-selective fading channels; Iterative algorithms

\section{Introduction}

The multipath channel and the additive noise are two important sources of distortion in wireless communication systems. Firstly, the channel impulse or frequency response provides information about the selectivity of the channel. Secondly, the noise is usually characterized by means of its comparison with the signal level by the signalto-noise ratio (SNR). The knowledge of these parameters (channel and noise) allows to design more accurately both transmitter and receiver. For instance, at the transmitter side, the constellation type and its size can be adapted according to the SNR level [1]. Yet, at the transmitter, the time-reversal method [2] can be performed, thanks to the channel impulse response. At the receiver side, many algorithms such as the LMMSE channel estimation [3] or the turbo-decoder [4] require the knowledge of the SNR, and an accurate channel state information (CSI) allows to perform a simple one-tap equalization in orthogonal frequency division multiplexing (OFDM) systems.

\footnotetext{
*Correspondence: vincent.savaux@ecam-rennes.com

1 ECAM Rennes-Louis de Broglie, Rennes, France

${ }^{2}$ IETR-Supélec, Rennes, France

Full list of author information is available at the end of the article
}

Some SNR estimation methods are proposed in [5-7] for single carrier systems that are being used in additive white Gaussian noise (AWGN) channels. The secondand fourth-order moment $\left(\mathrm{M}_{2} \mathrm{M}_{4}\right)$ estimator, firstly mentioned in [8], does not require any channel estimation. In addition, $\mathrm{M}_{2} \mathrm{M}_{4}$ has a low complexity. However, its efficiency is degraded in frequency-selective channels. The maximum likelihood (ML) estimator, whose developments are given in [9], offers a good efficiency, but has a prohibitive complexity in the case of frequencyselective channels. The minimum mean square error (MMSE) estimator, from which we derive our proposed method, requires the estimation of the transmission channel. In references $[5,10,11]$ only a theoretical expression of the MMSE can be found, but the authors do not propose any practical solution to reach it. Reference [10] covers the usual $\mathrm{M}_{2} \mathrm{M}_{4}, \mathrm{ML}$, and MMSE estimators in an OFDM case, and presents a method so as to estimate the SNR in frequency-selective channels. This latter method is based on the autocorrelation function given by the model of the channel (Rayleigh or Rice models). The authors of [11-13] also present SNR estimation methods for OFDM transmissions in frequency-selective channels. In order to avoid the need for the channel estimation, 
author in [11] proposes a method for a $2 \times 2$ multi-input and multi-output (MIMO) configuration which features a two pilot-symbols preamble and assumes that the channel coefficients are invariant over two consecutive carriers. Author in [12] also proposes a preamble-based method featuring two pilot symbols for the estimation of the noise variance. The SNR's estimation is performed, thanks to the combination of this noise estimation with the second moment order $\left(M_{2}\right)$ of the received signal. The methods of [11] and [12] require a two pilot-symbols preamble, which reduces the useful data rate of the transmission, especially if the preamble is regularly repeated. The authors of [13] present a SNR estimation based on the properties of the channel covariance matrix, estimated thanks to a one pilot-symbol preamble. This method is limited by the channel's insufficient statistics, which degrades the estimation performance.

The literature is very extensive concerning channel estimation. A wide range of usual channel estimation methods is based on ML [9], least square (LS) [14,15], or MMSE $[16,17]$. Here we focus on recursive and iterative methods. The recursive least square algorithm (RLS), described in [18] or [19], uses the estimations of the previous channel frequency response to perform the estimation of the current one. Similarly to the RLS principle, authors in $[20,21]$ propose a recursive MMSE method that does not require an $a$ priori need for channel statistics. In [22], the channel variations are tracked by employing the Kalman filter estimator. As presented in [23-26], the iterative channel estimation methods are combined with equalization, data detection, decoding, or even interference cancellation. In this case, a soft or hard feedback from the detection block to the estimator block performs an iterative channel and data estimation. The iterative expectation maximization (EM) algorithm $[27,28]$ has been developed so that the ML estimator is an appropriate tool in frequency-selective channels when the observed data are not complete, i.e., when the size of the observation is smaller than the vector to be estimated. An adaptation of this algorithm for both channel and noise estimation is presented in $[29,30]$, and joint iterative EM data detection and recursive channel tracking are proposed in [31]. However, when a preamble is used, the sizes of the observation and that of the vector of the channel frequency response to be estimated are the same, so the EM algorithm is not necessary. Furthermore, under this condition and considering a Gaussian channel, the ML estimator is equivalent to the usual LS estimator [32,33]. In [34], we proposed an MMSE-based iterative algorithm for both SNR and channel estimations. However, it was a theoretical approach, in which the channel covariance matrix was supposed to be known at the receiver.

In the present article, we propose an approach in which the channel covariance matrix is estimated at the receiver.
As a consequence, this paper is considered as an application of the theoretical approach developed in [34]. We estimate the SNR thanks to the MMSE criterion, which requires an estimation of the frequency-selective channel. Since we use the LMMSE method for the channel estimation, the noise variance is required. We clearly notice that one estimation feeds the other one. Then, it seems natural to propose an iterative algorithm. We show that it converges, thanks to a relevant choice of the initialization. Since we suppose no a priori CSI at the receiver, this algorithm is also valid for communications systems such as WiFi or LTE, broadcast systems featuring standards such as Digital Radio Mondiale DRM/DRM+ [35], or digital video broadcasting-terrestrial DVB-T [36]. Although we use the term iterative to describe our method, it differs from the usual iterative methods such as $[25,26,31]$ since it does not require a feedback from the data detection block.

The rest of this paper is organized as follows: Section 2 presents the used OFDM system model, the noise variance, and the SNR and channel estimations. The proposed algorithm is developed in Section 3, and we prove in Section 4 that it converges for a relevant choice of initialization. Simulations presented in Section 5 verify the convergence of the method. As for the SNR estimation, we compare our method to two others presented in the literature [12,13], the well-known $\mathrm{M}_{2} \mathrm{M}_{4}$ [10], and to the estimation performed in the perfect case [34]. The channel estimation performance is compared to the perfect one and to the usual LS. We draw our conclusions in Section 6.

\section{Background and system model \\ 2.1 Notations}

In the following, the normal font $x$ is used for scalar variables, the bold font $\mathbf{x}$ is used for vectors, and the underlined bold font $\underline{\mathbf{x}}$ for matrices. Furthermore, small letter $x$ refers to the variables in the time domain and capital letter $X$ to the variables in the frequency domain.

\subsection{System model}

We consider the transmission of OFDM symbols over a multipath channel. After the removal of the cyclic prefix (CP) and the discrete Fourier transform (DFT), we give the expression of the $n$ th-received OFDM symbol in the frequency domain as

$$
\mathbf{U}_{n}=\underline{\mathbf{C}}_{n} \mathbf{H}_{n}+\mathbf{W}_{n},
$$

where $\mathbf{U}_{n}=\left[U_{0, n}, \ldots, U_{M-1, n}\right]^{T}, \mathbf{H}_{n}=\left[H_{0, n}, \ldots, H_{M-1, n}\right]^{T}$, and $\mathbf{W}_{n}=\left[W_{0, n}, \ldots, W_{M-1, n}\right]^{T}$ denote the $M \times 1$ complex vectors of the received signal, the multipath channel, and the Gaussian white noise on the $n$th time slot, respectively. $M$ is the size of the DFT, which also tallies with the number of carriers per symbol in our model. The matrix $\underline{\mathbf{C}}_{n}$ is 
the $M \times M$ diagonal matrix of the transmitted signal containing the vector $\left[C_{0, n}, \ldots, C_{M-1, n}\right]$. Each $C_{m, n}$ is either a data element or a pilot, whose gain, phase, and position are perfectly known at both transmitter and receiver sides. In this article, the pilots are dedicated to channel estimation and noise variance estimation. We consider a pilot-preamble scheme with a sole OFDM pilot symbol and assume that the channel existing between two consecutive preambles is constant. In the rest of the paper, the pilot symbols are noted with the subscript $p$. Each component $H_{m, n}$ of the vector $\mathbf{H}_{n}$ is given by

$$
H_{m, n}=\sum_{l=0}^{L-1} h_{l, n} \exp \left(-2 j \pi \frac{m}{M} \tau_{l}\right),
$$

where $m$ denotes the subcarrier subscript, $L$ the length of the impulse response, and $h_{l, n}$ the zero-mean complex process of the $l$ th path of the channel. Each $\tau_{l}$ is the discrete expression of the delay. All $L$ paths are considered to be independent. We also assume a quasi-static channel, i.e., the coefficients $H_{m, n}$ are supposed to be invariant over a frame including a one pilot-symbol preamble and OFDM data symbols.

\subsection{Noise variance estimation}

We note $\sigma^{2}$ as the noise variance (or noise power) equal to $\sigma^{2}=E\left\{\left|W_{m, n}\right|^{2}\right\}$ in its scalar form or $\sigma^{2}=\frac{1}{M} E\left\{\left\|\mathbf{W}_{n}\right\|^{2}\right\}$ in the vectorial form. The matrix Frobenius norm noted $\|$.$\| is defined for a matrix \underline{\mathbf{A}}$ as $\|\underline{\mathbf{A}}\|^{2}=\operatorname{tr}\left(\underline{\mathbf{A A}}^{H}\right)$, with $\operatorname{tr}($.$) the trace and (.)^{H}$ the Hermitian transpose. In this article, we use the MMSE criterion to estimate the noise variance (see [9]) noted by $\hat{\sigma}^{2}$ and given by

$$
\hat{\sigma}^{2}=\frac{1}{M} E\left\{\left\|\mathbf{U}_{p}-\underline{\mathbf{C}}_{p} \hat{\mathbf{H}}_{p}\right\|^{2}\right\},
$$

where $\hat{\mathbf{H}}_{p}$ denotes the channel estimation performed on the pilot symbols. The index $p$ refers to the pilot preamble of a given frame. In practice, the expectation can only be approximated by the mean over a sufficiently large number of subcarriers, leading to $\hat{\sigma}^{2}=\frac{1}{M} \sum_{m=0}^{M-1} \mid U_{m, p}-$ $\left.C_{m, p} \hat{H}_{m, p}\right|^{2}$.

\subsection{SNR estimation}

The SNR noted $\rho$ is basically obtained from the secondorder moment $M_{2}$ of the received signal and the noise variance. Thus, $M_{2}=\frac{1}{M} E\left\{\left\|\mathbf{U}_{n}\right\|^{2}\right\}=P_{S}+\sigma^{2}$, with $P_{S}$ being the power of the useful transmitted signal. We then get the SNR, thanks to $\rho=\frac{M_{2}}{\sigma^{2}}-1$. In practice, we estimate the SNR $\hat{\rho}$ similarly:

$$
\hat{\rho}=\frac{\hat{M}_{2}}{\hat{\sigma}^{2}}-1,
$$

where $\hat{\sigma}^{2}$ is defined in (3) and $\hat{M}_{2}$ by $\hat{M}_{2}=\frac{1}{M}$ $\sum_{m=0}^{M-1}\left|U_{m, n}\right|^{2}$.

\subsection{Channel estimation}

The two basic channel estimation methods are the LS and LMMSE presented in $[14,16]$. Equation 5 gives the LS channel estimation

$$
\hat{\mathbf{H}}_{p}^{\mathrm{LS}}=\underline{\mathbf{C}}_{p}^{-1} \mathbf{U}_{p}=\mathbf{H}_{p}+\underline{\mathbf{C}}_{p}^{-1} \mathbf{W}_{p}
$$

The LS estimation is very simple but sensitive to the noise. Furthermore, this estimation cannot be used for the noise variance estimation in (3) since we obtain $\hat{\sigma}^{2}=0$ for $\hat{\mathbf{H}}_{p}=\hat{\mathbf{H}}_{p}^{\mathrm{LS}}$. Equation 6 gives the efficient LMMSE channel estimation as follows:

$$
\hat{\mathbf{H}}_{p}^{\mathrm{LMMSE}}=\underline{\mathbf{R}}_{H}\left(\underline{\mathbf{R}}_{H}+\sigma^{2}\left(\underline{\mathbf{C}}_{p} \underline{\mathbf{C}}_{p}^{H}\right)^{-1}\right)^{-1} \hat{\mathbf{H}}_{p}^{\mathrm{LS}},
$$

where $\underline{\mathbf{R}}_{H}$ denotes the channel covariance matrix. The LMMSE channel estimation is more efficient than that of the LS but requires a matrix inversion. Without loss of generality, we assume in the rest of the paper that $\forall m=$ $0, \ldots, M-1, C_{m, p}=1$ on a given preamble position $p$. Consequently, the pilot matrix $\underline{\mathbf{C}}_{p}$ is equal to the identity matrix noted $\underline{\mathbf{I}}$, which leads to $\hat{\mathbf{H}}_{p}^{\mathrm{LMMSE}}=\underline{\mathbf{R}}_{H}\left(\underline{\mathbf{R}}_{H}+\right.$ $\left.\sigma^{2} \underline{\mathbf{I}}\right)^{-1} \hat{\mathbf{H}}_{p}^{\text {LS }}$. As $\underline{\mathbf{R}}_{H}$ and $\sigma^{2}$ are usually unknown at the receiver, we propose an iterative algorithm for both noise variance and channel estimation.

\section{Proposed algorithm}

In this section, we present an iterative algorithm for the joint estimation of both the SNR and the frequencyselective channel. The algorithm is based on the MMSE criterion for both channel and SNR estimations. Indeed, we perform the noise variance estimation in (3), thanks to the efficient MMSE channel estimation, and the noise variance is used in Equation 6 for the LMMSE channel estimation. As each estimation feeds the other one, we propose an iterative algorithm, described in Figure 1. Since the channel covariance matrix used in the LMMSE estimation in Equation 6 is unknown, it must be estimated. We note this estimated covariance matrix $\underline{\tilde{\mathbf{R}}}_{H}$. Thus, the difficulty to overcome thereafter is the computation of a relevant channel covariance matrix. At the first iteration $i=1$, we only dispose of the LS channel estimation $\hat{\mathbf{H}}_{p}^{\mathrm{LS}}$, with which we compute the channel covariance matrix $\underline{\tilde{\mathbf{R}}}_{H}^{\mathrm{LS}}$ thanks to

$$
\underline{\tilde{\mathbf{R}}}_{H}^{\mathrm{LS}}=\hat{\mathbf{H}}_{p}^{\mathrm{LS}}\left(\hat{\mathbf{H}}_{p}^{\mathrm{LS}}\right)^{H} .
$$

For the first step of the algorithm, pointed out by the index $(i=1)$, the LMMSE channel estimation is performed as

$$
\hat{\mathbf{H}}_{p(i=1)}^{\mathrm{LMMSE}}=\underline{\tilde{\mathbf{R}}}_{H}^{\mathrm{LS}}\left(\underline{\tilde{\mathbf{R}}}_{H}^{\mathrm{LS}}+\hat{\sigma}_{(i=0)}^{2} \frac{\mathbf{I}}{)^{-1}} \hat{\mathbf{H}}_{p}^{\mathrm{LS}},\right.
$$

where $\hat{\sigma}_{(i=0)}^{2}$ is the initialization value of the algorithm. The noise variance is estimated as indicated in (3), with 


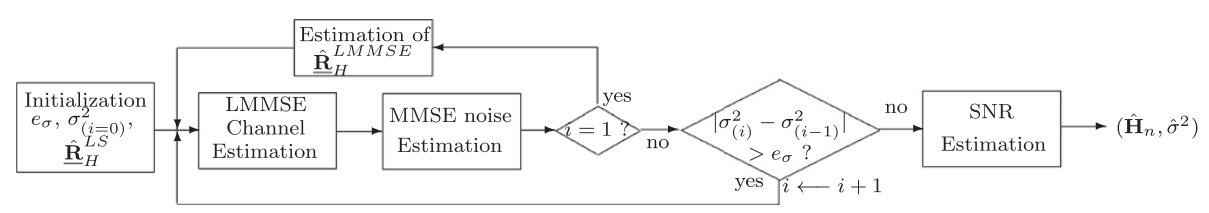

Figure 1 Block diagram of the proposed iterative algorithm.

$\hat{\mathbf{H}}=\hat{\mathbf{H}}_{p(i=1)}^{\mathrm{LMMSE}}$. Remembering that $\underline{\mathbf{C}}_{p}$ is equal to the identity matrix $\underline{\mathbf{I}}$, we have

$$
\begin{aligned}
\hat{\sigma}_{(i=1)}^{2}= & \frac{1}{M} E\left\{\left\|\mathbf{U}_{p}-\underline{\mathbf{C}}_{p} \hat{\mathbf{H}}_{p(i=1)}^{\mathrm{LMMSE}}\right\|^{2}\right\} \\
& \frac{1}{M} E\left\{\left\|\hat{\mathbf{H}}_{p}^{\mathrm{LS}}-\hat{\mathbf{H}}_{p(i=1)}^{\mathrm{LMMSE}}\right\|^{2}\right\} .
\end{aligned}
$$

If the algorithm keeps on computing with $\underline{\tilde{\mathbf{R}}}_{H}^{\mathrm{LS}}$, we prove in Appendix that $\left(\hat{\sigma}_{(i)}^{2}\right)$ converges to 0 . Under this condition, the algorithm enters into an endless loop. The reason is that $\underline{\tilde{\mathbf{R}}}_{H}^{\mathrm{LS}}$ is built thanks to one sole pilot symbol, and this makes it sensitive to the noise. The proposed solution is, for $i \geq 2$, to use the estimated covariance matrix $\underline{\tilde{\mathbf{R}}}_{H}^{\mathrm{LMMSE}}$ given by

$$
\underline{\tilde{\mathbf{R}}}_{H}^{\mathrm{LMMSE}}=\hat{\mathbf{H}}_{p(i=1)}^{\mathrm{LMMSE}}\left(\hat{\mathbf{H}}_{p(i=1)}^{\mathrm{LMMSE}}\right)^{H},
$$

instead of $\underline{\tilde{\mathbf{R}}}_{H}^{L S}$ in the following iterations. In Figure 1, the feedback from $\hat{\mathbf{H}}_{p}^{\mathrm{LMMSE}}$ is then valid only for $i=1$. For $i \geq 2$, the algorithm then follows these two steps:

$$
\begin{aligned}
\hat{\mathbf{H}}_{p(i)}^{\mathrm{LMMSE}} & =\underline{\tilde{\mathbf{R}}}_{H}^{\mathrm{LMMSE}}\left(\underline{\tilde{\mathbf{R}}}_{H}^{\mathrm{LMMSE}}+\hat{\sigma}_{(i-1)}^{2} \underline{\mathbf{I}}\right)^{-1} \hat{\mathbf{H}}_{p}^{\mathrm{LS}}, \\
\hat{\sigma}_{(i)}^{2} & =\frac{1}{M} E\left\{\left\|\hat{\mathbf{H}}_{p}^{\mathrm{LS}}-\hat{\mathbf{H}}_{p(i)}^{\mathrm{LMMSE}}\right\|^{2}\right\} .
\end{aligned}
$$

The characterization of the initialization $\hat{\sigma}_{(i=0)}^{2}$ will be discussed in Section 4. However, it is already obvious that $\hat{\sigma}_{(i=0)}^{2}$ must be strictly positive. Indeed, if its value is equal to 0 , then $\hat{\mathbf{H}}_{p(i=1)}^{\mathrm{LMMSE}}$ is equal to $\hat{\mathbf{H}}_{p}^{\mathrm{LS}}$ in (8).

Expressions (12) and (13) are obtained, thanks to a pilot matrix $\underline{\mathbf{C}}_{p}$ which is equal to the identity matrix $\underline{\mathbf{I}}$. In a general case, the pilot matrix $\underline{\mathbf{C}}_{p}$ (respectively, the pilot total energy) has to be taken into account in (12) and (13) respectively. A strictly positive threshold $e_{\sigma}$ is set and can be as small as needed; this entails that the algorithm proceeds iterations as long as $\left|\hat{\sigma}_{(i)}^{2}-\hat{\sigma}_{(i-1)}^{2}\right|>e_{\sigma}$. At last, if the final iteration is noted $\left(i_{0}\right)$, and if we use (4), the SNR is estimated from the noise variance thanks to

$$
\hat{\rho}=\frac{\hat{M}_{2}}{\hat{\sigma}_{\left(i_{0}\right)}^{2}}-1 .
$$

The algorithm given in the realistic case (considering an unknown channel covariance matrix) is summarized in Algorithm 1.

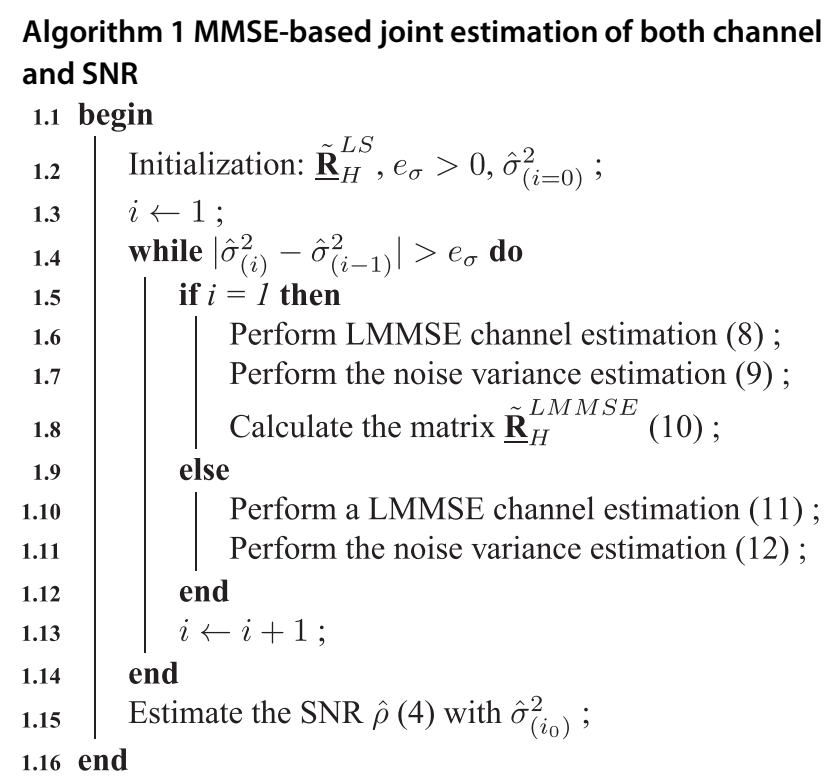

Figure 2 depicts the way our algorithm works. From the initialization $\hat{\sigma}_{(i=0)}^{2}$, the noise variance and channel estimations alternatively feed each other until the algorithm reaches its limit. Thus, step by step, the estimation $\left(\hat{\mathbf{H}}_{i, n}, \hat{\sigma}_{i}^{2}\right)$ comes closer to the limit characterized by the couple $\left(\hat{\mathbf{H}}_{i_{0}, n}, \hat{\sigma}_{i_{0}}^{2}\right)$. We also notice that this couple is different from the couple $\left(\mathbf{H}_{n}, \sigma^{2}\right)$, which characterizes the perfect estimation. This very low bias of estimation will be more precisely measured in Section 5 .

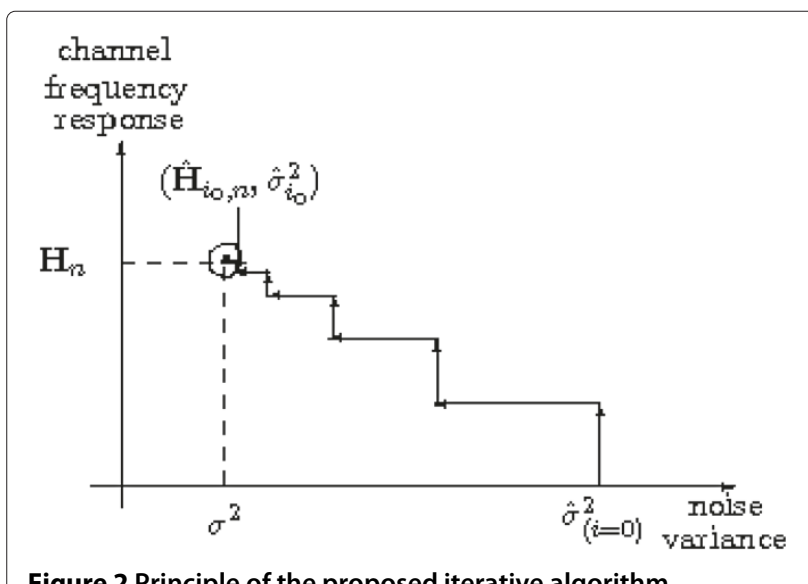

Figure 2 Principle of the proposed iterative algorithm. 


\section{Convergence of the algorithm in realistic case}

This section aims at proving that the algorithm converges in the realistic case (i.e., using an a priori unknown channel covariance matrix) if a relevant initialization $\hat{\sigma}_{(i=0)}^{2}$ is chosen. Thanks to the expressions (12) and (14), it is obvious that the channel estimation and the SNR converge, since the convergence of $\left(\hat{\sigma}_{(i)}^{2}\right)$ is established.

\subsection{Scalar expression of the sequence $\left(\hat{\sigma}_{(i)}^{2}\right)$}

As seen in (8) and (10) in Section 3, a first channel and noise variance estimation is performed using $\underline{\tilde{\mathbf{R}}}_{H}^{\mathrm{LS}}$. Indeed, the estimation of the covariance matrix $\underline{\tilde{\mathbf{R}}}_{H}^{\mathrm{LMMSE}}=$ $\hat{\mathbf{H}}_{p(i=1)}^{\mathrm{LMMSE}}\left(\hat{\mathbf{H}}_{p(i=1)}^{\mathrm{LMMSE}}\right)^{H}$ requires beforehand a LMMSE channel estimation. We prove in Appendix that if the algorithm is performed exclusively with $\underline{\mathbf{R}}_{H}^{\mathrm{LS}}$, this leads to a noise variance estimation which is equal to 0 . This justifies the substitution of $\underline{\tilde{\mathbf{R}}}_{H}^{\mathrm{LS}}$ by $\underline{\tilde{\mathbf{R}}}_{H}^{\mathrm{LMMSE}}$ for $i \geq 2$. The noise variance estimation at the $i$ th iteration (13) can be expressed as

$$
\begin{aligned}
\hat{\sigma}_{(i+1)}^{2}= & \frac{1}{M} E\left\{\left\|\hat{\mathbf{H}}_{p}^{\mathrm{LS}}-\hat{\mathbf{H}}_{p(i+1)}^{\mathrm{LMMSE}}\right\|^{2}\right\} \\
= & \frac{1}{M} \operatorname{tr}\left(\hat{\sigma}_{(i)}^{4}\left(\underline{\tilde{\mathbf{R}}}_{H}^{\mathrm{LMMSE}}+\hat{\sigma}_{(i)}^{2} \underline{\mathbf{I}}\right)^{-1}\left(\underline{\mathbf{R}}_{H}+\sigma^{2} \underline{\mathbf{I}}\right)\right. \\
& \left.\times\left(\underline{\tilde{\mathbf{R}}}_{H}^{\mathrm{LMMSE}}+\hat{\sigma}_{(i)}^{2} I\right)^{-1}\right)
\end{aligned}
$$

We express $\underline{\tilde{\mathbf{R}}}_{H}^{\text {LMMSE }}$, computed after the first iteration (11), as

$$
\begin{aligned}
\underline{\tilde{\mathbf{R}}}_{H}^{\mathrm{LMMSE}}= & \frac{1}{M} \hat{\mathbf{H}}_{p(i=1)}^{\mathrm{LMMSE}}\left(\hat{\mathbf{H}}_{p(i=1)}^{\mathrm{LMMSE}}\right)^{H} \\
= & \left(\underline{\tilde{\mathbf{R}}}_{H}^{\mathrm{LS}}\left(\underline{\tilde{\mathbf{R}}}_{H}^{\mathrm{LS}}+\hat{\sigma}_{(i=0)}^{2} \underline{\mathbf{I}}\right)^{-1} \hat{\mathbf{H}}_{p}^{\mathrm{LS}}\right) \\
& \times\left(\underline{\tilde{\mathbf{R}}}_{H}^{\mathrm{LS}}\left(\underline{\tilde{\mathbf{R}}}_{H}^{\mathrm{LS}}+\hat{\sigma}_{(i=0)}^{2} \underline{\mathbf{I}}\right)^{-1} \hat{\mathbf{H}}_{p}^{\mathrm{LS}}\right)^{H} \\
= & \underline{\tilde{\mathbf{R}}}_{H}^{\mathrm{LS}}\left(\underline{\tilde{\mathbf{R}}}_{H}^{\mathrm{LS}}+\hat{\sigma}_{(i=0)}^{2} \underline{\mathbf{I}}^{-1} \hat{\mathbf{H}}_{p}^{\mathrm{LS}}\left(\hat{\mathbf{H}}_{p}^{\mathrm{LS}}\right)^{H}\right. \\
& \times\left(\underline{\tilde{\mathbf{R}}}_{H}^{\mathrm{LS}}\left(\underline{\tilde{\mathbf{R}}}_{H}^{\mathrm{LS}}+\hat{\sigma}_{(i=0)}^{2} \underline{\mathbf{I}}^{-1}\right)^{H} .\right.
\end{aligned}
$$

For a sufficiently large value of $M$, we consider that $\frac{1}{M} \operatorname{tr}\left(\underline{\tilde{\mathbf{R}}}_{H}^{\mathrm{LS}}\right)=\frac{1}{M} \operatorname{tr}\left(\underline{\mathbf{R}}_{H}+\sigma^{2} \underline{\mathbf{I}}\right)$. Since the estimation of the noise variance is calculated thanks to the trace in (16), we make the assumption that $\underline{\mathbf{R}}_{H}^{\mathrm{LS}}=\hat{\mathbf{H}}_{p}^{\mathrm{LS}}\left(\hat{\mathbf{H}}_{p}^{\mathrm{LS}}\right)^{H}=\underline{\mathbf{R}}_{H}+\sigma^{2} \underline{\mathbf{I}}$ as a first approximation in (19). Remembering that $\underline{\mathbf{R}}_{H}+$ $\sigma^{2} \mathbf{I}$ is an Hermitian matrix leads us to consider that its inverse is also Hermitian, we get

$$
\begin{aligned}
\underline{\tilde{\mathbf{R}}}_{H}^{\mathrm{LMMSE}}= & \left(\underline{\mathbf{R}}_{H}+\sigma^{2} \underline{\mathbf{I}}\right)\left(\underline{\mathbf{R}}_{H}+\left(\sigma^{2}+\hat{\sigma}_{(i=0)}^{2}\right) \underline{\mathbf{I}}\right)^{-1} \\
& \times\left(\underline{\mathbf{R}}_{H}+\sigma^{2} \underline{\mathbf{I}}\right)\left(\underline{\mathbf{R}}_{H}+\left(\sigma^{2}+\hat{\sigma}_{(i=0)}^{2}\right) \underline{\mathbf{I}}\right)^{-1} \\
& \times\left(\underline{\mathbf{R}}_{H}+\sigma^{2} \underline{\mathbf{I}}\right) .
\end{aligned}
$$

As shown in [34], it is possible to obtain the diagonalized form of the expression (20). If we insert (20) into (16), we then get the scalar expression of the noise variance estimation:

$\hat{\sigma}_{(i+1)}^{2}=\frac{\hat{\sigma}_{(i)}^{4}}{M} \sum_{m=0}^{M-1} \frac{\left(\lambda_{m}+\sigma^{2}+\hat{\sigma}_{(i=0)}^{2}\right)^{4}\left(\lambda_{m}+\sigma^{2}\right)}{\left(\left(\lambda_{m}+\sigma^{2}\right)^{3}+\hat{\sigma}_{(i)}^{2}\left(\lambda_{m}+\sigma^{2}+\hat{\sigma}_{(i=0)}^{2}\right)^{2}\right)^{2}}$

$\Leftrightarrow \hat{\sigma}_{(i+1)}^{2}=\frac{\hat{\sigma}_{(i)}^{4}}{M} \sum_{m=0}^{M-1} \frac{\lambda_{m}+\sigma^{2}}{\left(\frac{\left(\lambda_{m}+\sigma^{2}\right)^{3}}{\left(\lambda_{m}+\sigma^{2}+\hat{\sigma}_{(i=0)}^{2}\right)^{2}}+\hat{\sigma}_{(i)}^{2}\right)^{2}}$,

where $\lambda_{m}$ are the eigenvalues of the matrix $\underline{\mathbf{R}}_{H}$. From (22) we remark that the choice of the initialization $\hat{\sigma}_{(i=0)}^{2}$ plays a key role in the convergence of the algorithm. Indeed, if $\hat{\sigma}_{(i=0)}^{2}$ is close to 0 , the term $\frac{\left(\lambda_{m}+\sigma^{2}\right)^{3}}{\left(\lambda_{m}+\sigma^{2}+\hat{\sigma}_{(i=0)}^{2}\right)^{2}}$ is then roughly equal to $\left(\lambda_{m}+\sigma^{2}\right)$. It is therefore equivalent to use the covariance matrix $\underline{\tilde{\mathbf{R}}}_{H}^{\mathrm{LS}}$ and the noise estimation then converges towards 0 . The solution is then to choose a value of $\hat{\sigma}_{(i=0)}^{2}$ that is arbitrarily large. Nevertheless, it is possible to characterize the initialization, thanks to the necessary condition given below. We define the function $f_{2}$ as $\hat{\sigma}_{(i+1)}^{2}=f_{2}\left(\hat{\sigma}_{(i)}^{2}\right)$. We set $x=\hat{\sigma}_{(i)}^{2} \geq 0$ so that the function $f_{2}(x)$ is

$$
f_{2}(x)=\frac{x^{2}}{M} \sum_{m=0}^{M-1} \frac{\lambda_{m}+\sigma^{2}}{\left(\frac{\left(\lambda_{m}+\sigma^{2}\right)^{3}}{\left(\lambda_{m}+\sigma^{2}+\hat{\sigma}_{(i=0)}^{2}\right)^{2}}+x\right)^{2}} .
$$

\subsection{Necessary condition for the convergence of the sequence $\left(\hat{\sigma}_{(i)}^{2}\right)$}

The limits of $f_{2}$ are $f_{2}(0)=0$ and $\lim _{x \rightarrow \infty} f_{2}(x)=M_{2}$. The derivative $f_{2}^{\prime}$ of $f_{2}(24)$ is positive,

$$
f_{2}^{\prime}(x)=\frac{2 x}{M} \sum_{m=0}^{M-1} \frac{\left(\lambda_{m}+\sigma^{2}\right) \frac{\left(\lambda_{m}+\sigma^{2}\right)^{3}}{\left(\lambda_{m}+\sigma^{2}+\hat{\sigma}_{(i=0)}^{2}\right)^{2}}}{\left(\frac{\left(\lambda_{m}+\sigma^{2}\right)^{3}}{\left(\lambda_{m}+\sigma^{2}+\hat{\sigma}_{(i=0)}^{2}\right)^{2}}+x\right)^{3}},
$$

so $f_{2}$ is growing in $[0,+\infty[$ and we can make the inclusion $f_{2}\left(\left[0, M_{2}\right]\right) \subset\left[0, M_{2}\right]$. Thus $f_{2}$ has at least a fixed point in the interval $\left[0, M_{2}\right]$. From (23), we know that 0 is an obvious fixed point of $f_{2}$. A necessary 
(but not sufficient) condition for $f_{2}$ to have other fixed points can be expressed as follows: there exists $x_{0} \geq 0$ such as $\max _{x}\left(f_{2}^{\prime}(x)\right)=f_{2}^{\prime}\left(x_{0}\right) \geq 1$, i.e., $f_{2}$ is above the first bisector. Then, $\hat{\sigma}_{(i=0)}^{2}$ can be adjusted in order to ensure this condition. If we note $f_{2_{m}}^{\prime}(x)$ as the function extracted from $f_{2}^{\prime}(x)$ such as $f_{2}^{\prime}(x)=\sum_{m=0}^{M-1} f_{2_{m}}^{\prime}(x)$, we have

$$
f_{2_{m}}^{\prime}(x)=\frac{1}{M} \frac{2 x\left(\lambda_{m}+\sigma^{2}\right) \frac{\left(\lambda_{m}+\sigma^{2}\right)^{3}}{\left(\lambda_{m}+\sigma^{2}+\hat{\sigma}_{(i=0)}^{2}\right)^{2}}}{\left(\frac{\left(\lambda_{m}+\sigma^{2}\right)^{3}}{\left(\lambda_{m}+\sigma^{2}+\hat{\sigma}_{(i=0)}^{2}\right)^{2}}+x\right)^{3}} .
$$

Let us note that $f_{2_{\min }}^{\prime}(x)$ as the function whose maximum that reached for $x=x_{0} \min$ is less important than any other maxima of the functions $f_{2_{m}}^{\prime}$ among the set $\left\{f_{2_{m}}^{\prime}\right\}$, $m=0, \ldots, M-1$. If we adjust $\hat{\sigma}_{(i=0)}^{2}$ so that $f_{2_{\min }}^{\prime}\left(x_{0 \min }\right) \geq$ 1 , then we fulfil the necessary condition, $f_{2}^{\prime}\left(x_{0 \mathrm{~min}}\right) \geq 1$. Indeed, if $f_{2_{\min }^{\prime}}^{\prime}\left(x_{0 \mathrm{~min}}\right) \geq 1$, then

$$
1 \leq f_{2_{\min }}^{\prime}\left(x_{0 \min }\right) \leq \frac{1}{M} \sum_{m=0}^{M-1} f_{2_{m}}^{\prime}\left(x_{0 \min }\right)=f_{2}^{\prime}\left(x_{0 \min }\right) .
$$

In order to find $x_{0 \mathrm{~min}}$, we calculate the second derivative $f_{2_{\min }}^{\prime \prime}$ of $f_{2_{\text {min }}}$ :

$f_{2_{m}}^{\prime \prime}(x)=\frac{1}{M} \frac{2\left(\lambda_{m}+\sigma^{2}\right) \frac{\left(\lambda_{m}+\sigma^{2}\right)^{3}}{\left(\lambda_{m}+\sigma^{2}+\hat{\sigma}_{(i=0)}^{2}\right)^{2}}\left(\frac{\left(\lambda_{m}+\sigma^{2}\right)^{3}}{\left(\lambda_{m}+\sigma^{2}+\hat{\sigma}_{(i=0)}^{2}\right)^{2}}-2 x\right)}{\left(\frac{\left(\lambda_{m}+\sigma^{2}\right)^{3}}{\left(\lambda_{m}+\sigma^{2}+\hat{\sigma}_{(i=0)}^{2}\right)^{2}}+x\right)^{4}}$.

The second derivative $f_{2_{\min }^{\prime \prime}}^{\prime \prime}$ is equal to 0 for $x_{0 \text { min }}=$ $\frac{1}{2} \frac{\left(\lambda_{m}+\sigma^{2}\right)^{3}}{\left(\lambda_{m}+\sigma^{2}+\hat{\sigma}_{(i=0)}^{2}\right)^{2}}$, so we get the maximum value of $f_{2_{\min }^{\prime}}^{\prime}$ :

$$
f_{2_{\text {min }}}^{\prime}\left(x_{0 \text { min }}\right)=\frac{8}{27} \frac{\left(\lambda_{m}+\sigma^{2}+\hat{\sigma}_{(i=0)}^{2}\right)^{2}}{\left(\lambda_{m}+\sigma^{2}\right)^{2}} .
$$

Whatever the values of $\sigma^{2}$ and $\hat{\sigma}_{(i=0)}^{2}, f_{2_{\min }}^{\prime}\left(x_{0 \text { min }}\right)$ are minimum for $\lambda_{m}=\lambda_{\max }$, with $\lambda_{\max }$ the maximum eigenvalue of $\underline{\mathbf{R}}_{H}$, we can then minimize $\hat{\sigma}_{(i=0)}^{2}$ :

$$
\begin{gathered}
\frac{8}{27} \frac{\left(\lambda_{\max }+\sigma^{2}+\hat{\sigma}_{(i=0)}^{2}\right)^{2}}{\left(\lambda_{\max }+\sigma^{2}\right)^{2}} \geq 1 \\
\Leftrightarrow \hat{\sigma}_{(i=0)}^{2} \geq\left(\sqrt{\frac{27}{8}}-1\right)\left(\lambda_{\max }+\sigma^{2}\right) .
\end{gathered}
$$

The necessary condition $\max _{x}\left(f_{2}^{\prime}(x)\right) \geq 1$ is fulfilled for $\hat{\sigma}_{(i=0)}^{2} \geq\left(\sqrt{\frac{27}{8}}-1\right)\left(\lambda_{\max }+\sigma^{2}\right)$. Since $\lambda_{\max }$ and $\sigma^{2}$ are unknown, the condition is necessary but not sufficient so as to assess that $f_{2}$ has a fixed point that is different from 0 .
However, $\lambda_{\max }$ is, by definition, the maximum eigenvalue of the channel covariance matrix, so $\lambda_{\max } \geq \frac{1}{M} \sum_{m=0}^{M-1} \lambda_{m}$. Furthermore, as $M_{2}=\frac{1}{M} \sum_{m=0}^{M-1} \lambda_{m}+\sigma^{2}$, so thanks to (30), we can minimize $\hat{\sigma}_{(i=0)}^{2}$ and get

$$
\hat{\sigma}_{(i=0)}^{2} \geq\left(\sqrt{\frac{27}{8}}-1\right) M_{2} .
$$

\subsection{Sufficient condition for the convergence of the sequence $\left(\hat{\sigma}_{(i)}^{2}\right)$}

The lower bound (31) satisfies the necessary condition $f_{2}^{\prime} \geq 1$. Thus, this entails that $f_{2}$ has a fixed point which is different from 0 . In order to give a sufficient condition, the initialization value $\hat{\sigma}_{(i=0)}^{2}$ has to be set equal to $\Lambda M_{2}$, with $\Lambda>>1$. Indeed, for all $x \in\left[0, M_{2}\right]$, we have $\lim _{\hat{\sigma}_{(i=0)}^{2} \rightarrow+\infty} f(x)=M_{2}$, so it is possible to find $\hat{\sigma}_{(i=0)}^{2}$ such as $f(x)>x$. Given that $\lim _{x \rightarrow+\infty} f(x)=M_{2}$, we deduce that a fixed point different from 0 exists for a well-chosen initialization $\hat{\sigma}_{(i=0)}^{2}=\Lambda M_{2}$. However, the previous development only proves the existence of a sufficient condition on $\hat{\sigma}_{(i=0)}^{2}$ for the convergence of $\left(\hat{\sigma}_{(i)}^{2}\right)$ to a non-null limit but it does not give a precise characterization of $\hat{\sigma}_{(i=0)}^{2}$. In order to get a suitable value of $\hat{\sigma}_{(i=0)}^{2}$, the receiver should test some initialization values (e.g., thanks to an abacus) until it finds the expected one, as depicted on Figure 3. The latter illustrates the shape of two examples of $f_{2}$ : one with a relevant initialization $\hat{\sigma}_{(i=0)}^{2}=10 M_{2}$ (we see a fixed point that is different from 0 ) and one with an initialization which does not match the necessary condition ( 0 is the sole fixed point). We actually observe that if $\hat{\sigma}_{(i=0)}^{2}$ is not chosen as being large enough, then $\hat{\sigma}_{(i)}^{2}$ converges to 0 .

However, the choice of a relevant value $\Lambda$ is not obvious. Indeed, since the channel frequency response and the

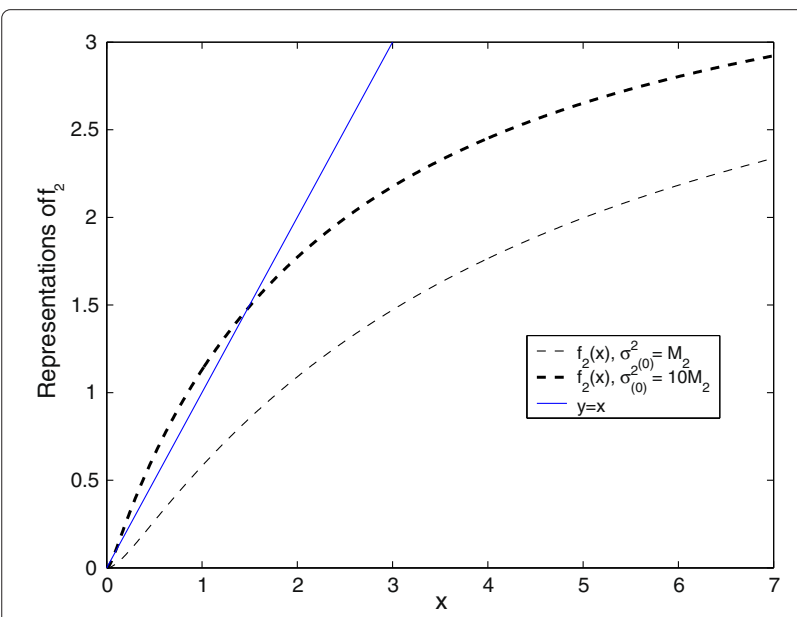

Figure 3 Shape of $f_{2}$ in two different cases compared with $y=x$. 
noise variance can take an infinite number of values, the design of an abacus of $f_{2}$ is computationally prohibitive. Furthermore, we assume that the receiver has no $a$ priori knowledge of the set of the parameters $\left\{\lambda_{m}, \sigma^{2}\right\}$, which makes the choice of the optimal initialization impossible. In order to overcome the issue of the complexity of the choice of $\hat{\sigma}_{(i=0)}^{2}$, we propose in Section 4.4 a simple characterization which does not require any abacus.

\subsection{Optimal choice of the initialization $\hat{\sigma}_{(i=0)}^{2}$}

The conditions on $\hat{\sigma}_{(i=0)}^{2}$ given in previous Section 4.3 are either not relevant enough $\left(\hat{\sigma}_{(i=0)}^{2}=\Lambda M_{2}\right.$ with $\left.\Lambda>>1\right)$, or too complex (use of abacus of $f_{2}$ ). Here, we propose a simple characterization of $\hat{\sigma}_{(i=0)}^{2}$ made thanks to the noise variance estimation $\hat{\sigma}^{2}$, which was performed on the last frame. If we note $\mathcal{F}$ the index of the current frame, the proposed method is as follows:

- For the first frame $\mathcal{F}=1$, perform the algorithm thanks to the arbitrary initialization $\hat{\sigma}_{(i=0)}^{2}=\Lambda M_{2}$ chosen with the sufficient condition $\Lambda>>1$.

- For $\mathcal{F}>1$, get the noise variance $\hat{\sigma}^{2}$ and the eigenvalues of the channel covariance matrix $\underline{\mathbf{R}}_{H}^{\mathrm{LMMS}}$ (11), estimated at the previous frame $\mathcal{F}-1$.

- Considering the expression of $f_{2}$ given in (23), look for $\hat{\sigma}_{(i=0) \text {,opt }}^{2}$ so that

$$
\frac{\hat{\sigma}^{4}}{M} \sum_{m=0}^{M-1} \frac{\lambda_{m}+\hat{\sigma}^{2}}{\left(\frac{\left(\lambda_{m}+\hat{\sigma}^{2}\right)^{3}}{\left(\lambda_{m}+\hat{\sigma}^{2}+\hat{\sigma}_{(i=0), o p t}^{2}\right)^{2}}+\hat{\sigma}^{2}\right)^{2}}-\hat{\sigma}^{2}=0 .
$$

The direct solving of (32) is very complex, but in practice, the receiver can use a simple binary search algorithm to approach the optimal solution. This optimal solution $\hat{\sigma}_{(i=0) \text {,opt }}^{2}$ can then be found at the frame $\mathcal{F}$.

For a relevant choice of the initialization $\hat{\sigma}_{(i=0)}^{2}$, we have given a sufficient condition so that the algorithm converges to a non-null solution. Additionally, an optimal value of $\hat{\sigma}_{(i=0)}^{2}$ can be found, which allows the convergence to take place at the expected noise and channel values. Section 5 depicts the performance of our algorithm and finally shows that the estimated couple $\left(\hat{\sigma}^{2}, \hat{\mathbf{H}}_{n}\right)$ is close to the perfect estimation one $\left(\sigma^{2}, \mathbf{H}_{n}\right)$.

\section{Simulations results}

This section aims at confirming, by means of simulations, the theoretical results developed in the previous sections. Furthermore, it characterizes the algorithm performance, such as the speed of convergence, the bias of the noise variance estimation, or the bit error rate (BER), thanks to the proposed channel estimation compared to the perfect one. The simulation parameters are based on the Digital Radio Mondiale (DRM/DRM+) standard [35], designed for the digital audio broadcasting over the current AM/FM frequency bands. We consider a 201subcarrier OFDM modulation with a sampling frequency equal to $12 \mathrm{kHz}$. Each OFDM frame is composed of 20 symbols, each symbol being filled with data symbols from a 16-QAM constellation. The added CP featuring a $T_{\mathrm{CP}}$ duration of $2.66 \mathrm{~ms}$ is supposed to avoid the intersymbol interferences, i.e., it is longer than the maximum delay of the channel. Although the DRM standard recommends a distribution of the pilot tones in staggered rows in the OFDM frame, we considered a preamble distribution for the purpose for our method. As previously mentioned, each preamble is composed of one pilot symbol only. We consider the US Consortium channel model, also described in the DRM standard. It is a four-path channel in which the maximum delay is $\tau_{\max }=2.2 \mathrm{~ms}$ and the maximum Doppler spread is equal to $2 \mathrm{~Hz}$. Here, the channel is supposed to be quasi-static, which means that it varies very slowly during a frame duration. In the following, the term 'perfect case' refers to the algorithm proposed in [34], in which the channel covariance matrix is supposed to be known at the receiver, whereas 'practical case' refers to the proposed algorithm.

\subsection{Convergence of the noise variance estimation}

Figure 4 depicts the noise variance estimation as a function of the iteration number $i$. The estimation in the practical case is compared to that of the perfect case and the real value of the noise variance. Furthermore, two values of SNR are considered: $\rho=0 \mathrm{~dB}$ for the upper curves and $\rho=10 \mathrm{~dB}$ for the lower curves. The initialization value $\hat{\sigma}_{(i=0)}^{2}$ in the perfect case is equal to 2 . In the practical

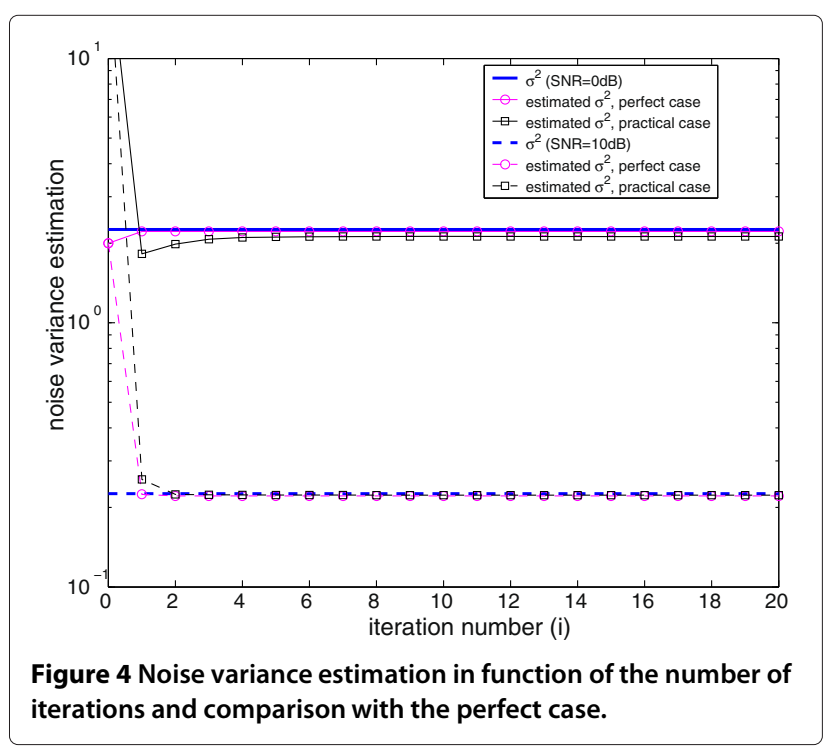


case, it is equal to $\hat{\sigma}_{(i=0)}^{2}=20 M_{2}$. The curves are obtained by means of an average made over 4,000 simulation runs.

For iterations $i \geq 1$, we remark that the sequence $\left(\hat{\sigma}_{(i)}^{2}\right)$ is monotonous and converges to a single non-null value, which verifies the theoretical developments given in Section 4. We also observe a fast convergence to the single limit, which will be confirmed in Section 5.2. Figure 4 characterizes the noise variance estimation thanks to the normalized bias $\beta$ calculated by $\beta=\left|\left(\hat{\sigma}_{(i)}^{2}\right)-\sigma^{2}\right| / \sigma^{2}, \beta$ being averaged over 4,000 runs. Expressed in percentage, the bias of the proposed estimation is equal to $5.9 \%$ for $\rho=0 \mathrm{~dB}$ and $1.2 \%$ for $\rho=10 \mathrm{~dB}$. These results are very close to the estimation performed in the perfect case.

\subsection{Speed of convergence of the algorithm}

Figure 5 shows the values of the difference $\left|\hat{\sigma}_{(i)}^{2}-\hat{\sigma}_{(i-1)}^{2}\right|$ versus the number of iterations for $i \geq 2$, for both the perfect and the practical cases. Simulations are performed with $\rho=10 \mathrm{~dB}$ and the initialization value $\hat{\sigma}_{(i=0)}^{2}=2$ in the perfect case and $\hat{\sigma}_{(i=0)}^{2}=20 M_{2}$ in the practical case. These curves characterize the required number of iterations to get an expected value of threshold $e_{\sigma}$.

For example, in order to reach a fixed value $e_{\sigma}=0.01$, three iterations are required in the practical case and two in the perfect case. For $e_{\sigma}=0.0001$, seven iterations are required in the practical case and three in the perfect case. These results confirm the high-speed convergence of the algorithm.

\subsection{Comparison of SNR estimation with other methods}

Figures 6 and 7 display the normalized MSE (NMSE) of the SNR estimation of the proposed method compared to the perfect case and three existing methods: Ren's method [12], Xu's method [13], and the usual $\mathrm{M}_{2} \mathrm{M}_{4}$ [10]. Ren's method requires a two pilot-symbols preamble

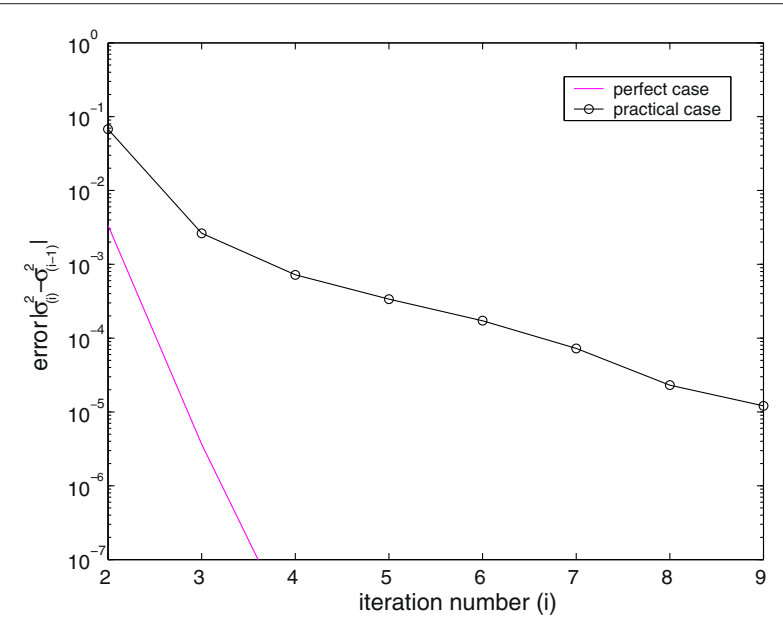

Figure 5 Error $\left|\hat{\sigma}_{(i)}^{2}-\hat{\sigma}_{(i-1)}^{2}\right|$ in function of the iteration number $i$.

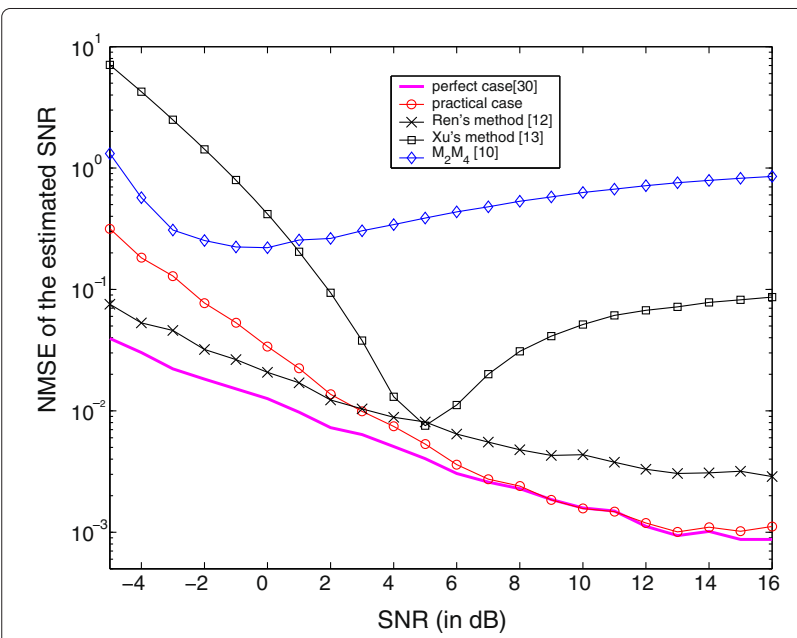

Figure 6 Comparison of NMSE of SNR estimation of the proposed method with existing methods at condition $\hat{\boldsymbol{\sigma}}_{(\boldsymbol{i}=\mathbf{0})}^{\mathbf{2}}=\mathbf{2 0} \mathbf{M}_{\mathbf{2}}$. NMSE of the SNR estimation of the proposed method compared to the two existing methods; $\hat{\sigma}_{(i=0)}^{2}$ is chosen thanks to the sufficient condition $\hat{\sigma}_{(i=0)}^{2}=20 M_{2}$.

in order to avoid the effect of the frequency-selective channel. The noise variance estimation is then given by $\hat{\sigma}^{2}=0.5\left\|\mathbf{U}_{p+1}-\mathbf{U}_{p}\right\|^{2}=0.5\left\|\mathbf{W}_{p+1}-\mathbf{W}_{p}\right\|^{2}$, and the SNR is computed thanks to (4). Xu's method requires a one pilot-symbol preamble in order to compute the covariance matrix of the channel. The noise variance estimation is made thanks to the subspace of the eigenvalues which includes only the noise samples. The $\mathrm{M}_{2} \mathrm{M}_{4}$ method directly computes the SNR estimation, thanks to the second moment order $M_{2}$ and the fourth moment

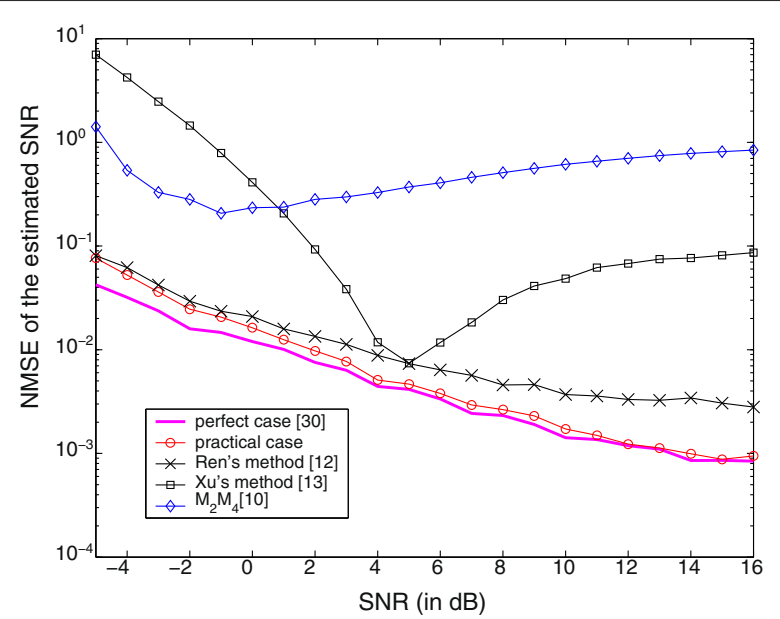

Figure 7 Comparison of NMSE of SNR estimation of the proposed method with existing methods choosing $\hat{\boldsymbol{\sigma}}_{(\mathbf{0})}^{2}$. NMSE of the SNR estimation of the proposed method compared to two existing methods; $\hat{\sigma}_{(0)}^{2}$ is chosen thanks to the proposed characterization of Section 4.4 . 
order $M_{4}$ of the received signal. For a 16-QAM constellation in Rayleigh fading channels, authors in [10] give the estimation of the SNR as $\hat{\rho}=\left(\sqrt{M_{4}-2 M_{2}^{2}}\right) /\left(0.8 M_{2}^{2}-\right.$ $\sqrt{M_{4}-2 M_{2}^{2}}$ ). Figures 6 and 7 also compare the SNR estimation performed with two different initializations. In Figure 6, we use the sufficient condition $\hat{\sigma}_{(i=0)}^{2}=20 M_{2}$ for each frame. In Figure 7, the initialization step $\hat{\sigma}_{(i=0)}^{2}=$ $20 M_{2}$ is used for the first frame $\mathcal{F}=1$, and then $\hat{\sigma}_{(i=0)}^{2}$ is updated thanks to the proposed method presented in Section 4.4. When Figure 6 or 7 is considered, we now consider $i_{0}=7$ iterations. In the perfect case, the initialization value is $\hat{\sigma}_{(i=0)}^{2}=0.1$, and the number of iterations is $i_{0}=3$. The NMSE given by NMSE $=E\left\{|\hat{\rho}-\rho|^{2} / \rho^{2}\right\}$ is approximated and simulated, thanks to an average made over 200,000 samples.

As mentioned in [10], we remark that the performance of the $\mathrm{M}_{2} \mathrm{M}_{4}$ method is degraded in Rayleigh channels, which is the case here. Whatever the SNR is, the proposed method outperforms the one from Xu's. In Figure 6, the performance of the algorithm is degraded compared to that obtained with the Ren's method for low SNR values $(<3 \mathrm{~dB})$. It confirms that for low values of SNR, $\hat{\sigma}_{(i=0)}^{2}$ is not large enough compared to the value of the noise variance $\sigma^{2}$. However, when the algorithm is used with an updated initialization (Figure 7), the method outperforms Ren's one whatever the SNR is, and the SNR gap with the perfect case is less than $1 \mathrm{~dB}$ from $S N R=0 \mathrm{~dB}$. This proves the efficiency of the proposed algorithm and the validity of the improvement with regard to the choice of $\hat{\sigma}_{(i=0)}^{2}$, when performed with an update in each frame. Furthermore, our method requires only one pilot-symbol preamble while Ren's requires two. We conclude that we can improve both the rate of the required pilots and the efficiency of the estimation.

\subsection{Channel estimation}

Figure 8 illustrates the BER of the proposed method as a function of the SNR over a relevant span (from 0 to 32 $\mathrm{dB})$. The estimation is compared to the ones performed in the perfect case, perfect estimation, and the usual LS. We remind that the channel is the four-path US Consortium from the DRM standard [35] and the constellation is a 16-QAM. The initialization is chosen as previously, i.e., $\hat{\sigma}_{(i=0)}^{2}=0.1$ in the perfect case and $\hat{\sigma}_{(i=0)}^{2}=20 M_{2}$ with an update in the practical case. The BER curves are performed by the means of simulating a $2.5 \times 10^{6}$ bits transmission. As mentioned in Section 2, the channel is quasi-static, so it is considered as being invariant on each frame of 20 OFDM symbols, where the first one is a pilot-symbol preamble.

We observe on Figure 8 that the channel estimation converges to a value that is close to the perfect estimation.

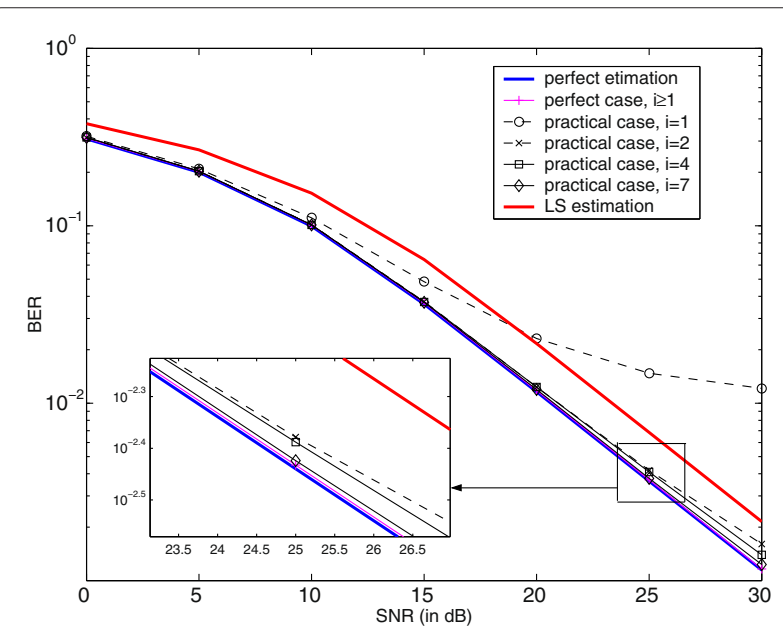

Figure 8 Proposed method BER versus SNR and comparison with the perfect estimation, perfect case, and LS.

Indeed, zooming around $\mathrm{SNR}=25 \mathrm{~dB}$, we can see that the gap between the perfect channel estimation and our method is less than $0.2 \mathrm{~dB}$ after seven iterations. It confirms the high speed of convergence and illustrates the efficiency of the channel estimation algorithm.

\section{Conclusions}

In this article, we presented a practical algorithm for a joint and iterative MMSE-based estimation of the SNR and the frequency-selective channel in an OFDM context. The SNR is estimated, thanks to the MMSE noise variance estimation combined with the second moment order of the signal, and the channel, thanks to the LMMSE method. Since each estimation requires the other one, the algorithm is iterative, as proposed in [34] for a theoretical case in which the channel covariance matrix is supposed to be known at the receiver. However, we considered in this paper a practical case which assumes that the channel covariance matrix is a priori unknown at the receiver side. We theoretically proved that for a well-chosen initialization value, the algorithm converges. Furthermore, simulations showed that the proposed method has a very good quality of estimation for both the SNR and the channel frequency response. Compared to existing methods, the algorithm improves the ratio between the required number of pilots and the efficiency of the SNR estimation. Further works will concern the application of the proposed algorithm to the domain of the cognitive radio, in particular, for free bands detection.

\section{Appendix}

We prove the convergence towards 0 of the algorithm when it is performed with the channel covariance matrix $\underline{\tilde{\mathbf{R}}}_{H}^{\mathrm{LS}}$. It justifies the substitution of this matrix by $\underline{\tilde{\mathbf{R}}}_{H}^{\mathrm{LMMSE}}$ 
for $i \geq 2$. We give the expression of the noise variance (13) using the developments made for the theoretical case [34]:

$$
\begin{aligned}
\hat{\sigma}_{(i+1)}^{2}= & \frac{1}{M} E\left\{\left\|\hat{\mathbf{H}}_{p}^{\mathrm{LS}}-\hat{\mathbf{H}}_{p(i+1)}^{\mathrm{LMMSE}}\right\|^{2}\right\} \\
= & \frac{1}{M} \operatorname{tr}\left(\hat{\sigma}_{(i)}^{4}\left(\underline{\tilde{\mathbf{R}}}_{H}^{\mathrm{LS}}+\hat{\sigma}_{(i)}^{2} \mathbf{I}\right)^{-1}\left(\underline{\mathbf{R}}_{H}+\sigma^{2} \underline{\mathbf{I}}\right)\right. \\
& \left.\times\left(\left(\underline{\tilde{\mathbf{R}}}_{H}^{\mathrm{LS}}+\hat{\sigma}_{(i)}^{2} I\right)^{-1}\right)^{H}\right) .
\end{aligned}
$$

We remind that for a large value $M$, we have $\frac{1}{M} \operatorname{tr}\left(\underline{\tilde{\mathbf{R}}}_{H}^{\mathrm{LS}}\right)=$ $\frac{1}{M} \operatorname{tr}\left(\underline{\mathbf{R}}_{H}+\sigma^{2} \underline{\mathbf{I}}\right)$, so we will consider, in a first approximation, that $\underline{\tilde{\mathbf{R}}}_{H}^{\mathrm{L} S}=\underline{\mathbf{R}}_{H}+\sigma^{2} \underline{\mathbf{I}}$ in order to develop (34)

$$
\begin{aligned}
\hat{\sigma}_{(i+1)}^{2}= & \frac{1}{M} \operatorname{tr}\left(\hat{\sigma}_{(i)}^{4}\left(\underline{\mathbf{R}}_{H}+\left(\hat{\sigma}_{(i)}^{2}+\sigma^{2}\right) \underline{\mathbf{I}}\right)^{-1}\left(\underline{\mathbf{R}}_{H}+\sigma^{2} \underline{\mathbf{I}}\right)\right. \\
& \left.\times\left(\left(\underline{\mathbf{R}}_{H}+\left(\hat{\sigma}_{(i)}^{2}+\sigma^{2}\right) \underline{\mathbf{I}}\right)^{-1}\right)^{H}\right) .
\end{aligned}
$$

With $\underline{\mathbf{R}}_{H}$ being an Hermitian matrix, we use the same diagonalization property as in [34] for the expression (35), and we finally find the scalar form of (35):

$$
\hat{\sigma}_{(i+1)}^{2}=\frac{\hat{\sigma}_{(i)}^{4}}{M} \sum_{m=0}^{M-1} \frac{\lambda_{m}+\sigma^{2}}{\left(\lambda_{m}+\sigma^{2}+\hat{\sigma}_{(i)}^{2}\right)^{2}},
$$

where $\lambda_{m}$ are the eigenvalues of the covariance matrix $\underline{\mathbf{R}}_{H}$. If we note $x=\hat{\sigma}_{(i)}^{2}$, the sequence $\left(\hat{\sigma}_{(i+1)}^{2}\right)$ is built from a function $f_{1}$ so that

$$
f_{1}(x)=\frac{x^{2}}{M} \sum_{m=0}^{M-1} \frac{\lambda_{m}+\sigma^{2}}{\left(\lambda_{m}+\sigma^{2}+x\right)^{2}},
$$

with $x \in[0,+\infty$. The proof of the convergence towards zero of the sequence $\left(\hat{\sigma}_{(i+1)}^{2}\right)$ in (36) is based on the fixed point theorem, i.e., we show that the only solution to the equation $f_{1}(x)=x$ is 0 . The limits of $f_{1}$ are $f_{1}(0)=0$ and $\lim _{x \rightarrow \infty} f_{1}(x)=\frac{1}{M} \sum_{m=0}^{M-1}\left(\lambda_{m}+\sigma^{2}\right)=M_{2}$. Furthermore, the derivative of $f_{1}$

$$
f_{1}^{\prime}(x)=\frac{2 x}{M} \sum_{m=0}^{M-1} \frac{\left(\lambda_{m}+\sigma^{2}\right)^{2}}{\left(\lambda_{m}+\sigma^{2}+x\right)^{3}},
$$

is positive for $x \in\left[0,+\infty\left[\right.\right.$, so $f_{1}$ is growing on this interval. We then deduce the inclusion $f_{1}\left(\left[0,+\infty[) \subset\left[0, M_{2}\right]\right.\right.$ and so $f_{1}\left(\left[0, M_{2}\right]\right) \subset\left[0, M_{2}\right]$. Thus $f_{1}$ has at least one fixed point on $\left[0, M_{2}\right]$. As $f_{1}$ is growing on $\left[0, M_{2}\right]$, we conclude that the sequence $\left(\hat{\sigma}_{(i+1)}^{2}\right)$ converges to one of the fixed point of $f_{1}$. An obvious fixed point of $f_{1}$ is 0 , since $f_{1}(0)=0$. We now prove that 0 is the sole fixed point of $f_{1}$ on $\left[0, M_{2}\right]$. To this end, we show that $f_{1}^{\prime}(x)<1$, which is equivalent to $\left(f_{1}(x)-x\right)^{\prime}<0$. We define the functions $f_{1_{m}}(x)$ extracted from $f_{1}(x)$ so that $f_{1}(x)=\frac{1}{M} \sum_{m}^{M-1} f_{1_{m}}(x)$ :

$$
f_{1_{m}}(x)=\frac{x^{2}\left(\lambda_{m}+\sigma^{2}\right)}{\left(\lambda_{m}+\sigma^{2}+x\right)^{2}} .
$$

Since $f_{1}$ is defined by a sum, we also have for the derivative $f_{1}^{\prime}(x)=\frac{1}{M} \sum_{m}^{M-1} f_{1_{m}}^{\prime}(x)$, with:

$$
f_{1_{m}}^{\prime}(x)=\frac{2 x\left(\lambda_{m}+\sigma^{2}\right)^{2}}{\left(\lambda_{m}+\sigma^{2}+x\right)^{3}} .
$$

For any value of $m=0,1, \ldots, M-1$ and $x \geq 0, f_{1_{m}}^{\prime}(x) \geq$ 0 , so we can apply the following triangle inequality on the derivate of $f_{1}$ :

$$
\max _{x}\left(f_{1}^{\prime}(x)\right) \leq \frac{1}{M} \sum_{m=0}^{M-1} \max _{x, m}\left(f_{1_{m}}^{\prime}(x)\right) .
$$

For $m=0,1, \ldots, M-1$, we find the maximum of $f_{1_{m}}^{\prime}(x)$, thanks to a second derivation so that

$$
f_{1_{m}}^{\prime \prime}(x)=\frac{2\left(\lambda_{m}+\sigma^{2}\right)^{2}\left(\lambda_{m}+\sigma^{2}-2 x\right)}{\left(\lambda_{m}+\sigma^{2}+x\right)^{4}} .
$$

The second derivative of $f_{1_{m}}(x)$ in (42) is null for $x=$ $\frac{1}{2}\left(\lambda_{m}+\sigma^{2}\right)$, so we find, thanks to expression (40)

$$
\max _{x}\left(f_{1_{m}}^{\prime}(x)\right)=f_{1_{m}}^{\prime}\left(x=\frac{1}{2}\left(\lambda_{m}+\sigma^{2}\right)\right)=\frac{8}{27} .
$$

Equation 43 shows that for any value of $m=0$, $1, \ldots, M-1$, the maximum of $f_{1_{m}}^{\prime}$ is equal to $\frac{8}{27}$, so the triangle inequality is simplified:

$$
\max _{x}\left(f_{1}^{\prime}(x)\right) \leq \frac{8}{27}
$$

which then proves that $f_{1}^{\prime}(x)<1$, i.e., $f$ has only one fixed point equal to 0 . Figure 9 displays an example of $f_{1}(x)$ and

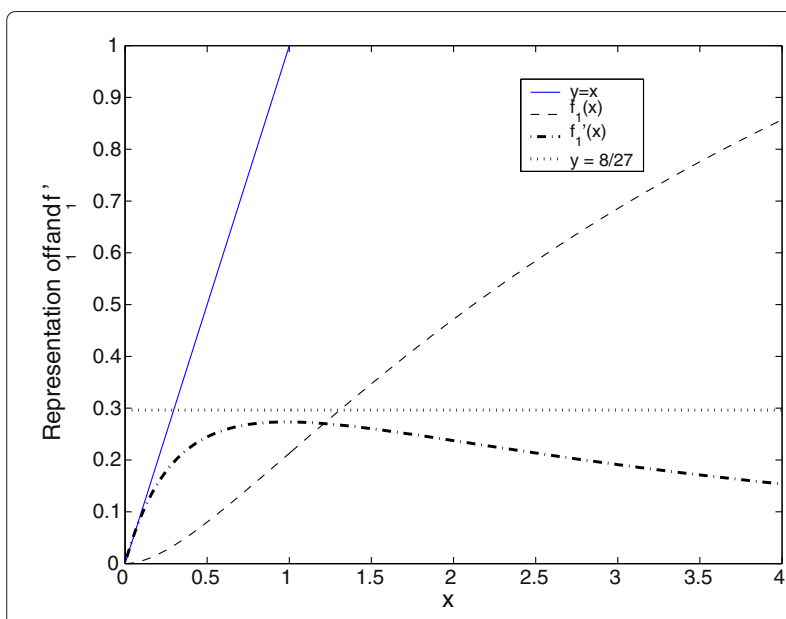

Figure 9 Shape of $f_{1}(x), f_{1}^{\prime}(x)$ compared with $y=x$ and $y=8 / 27$. 
$f_{1}^{\prime}(x)$. We conclude that if the algorithm is performed with the covariance matrix $\underline{\tilde{\mathbf{R}}}_{H}^{\mathrm{LS}}$, then the sequence $\left(\hat{\sigma}_{(i)}^{2}\right)$ converges to 0 and the algorithm enters into a endless loop, whatever the value of the initialization $\hat{\sigma}_{(0)}^{2}$ is.

\section{Competing interests}

The authors declare that they have no competing interests.

\author{
Author details \\ ${ }^{1}$ ECAM Rennes-Louis de Broglie, Rennes, France. ${ }^{2}$ IETR-Supélec, Rennes, \\ France. ${ }^{3}$ Zodiac Data Systems, Caen, France.
}

Received: 26 March 2013 Accepted: 17 June 2013

Published: 12 July 2013

\section{References}

1. T Keller, L Hanzo, Adaptive multicarrier modulation: a convenient framework for time-frequency processing in wireless communications. Proc. IEEE. 88(5), 611-640 (2000)

2. IH Naqvi, P Besnier, GE Zein, Effects of time variant channel on a time reversal UWB system, in Proceedings of the IEEE Global Telecommunications Conference, GLOBECOM 2009, Honolulu, 30 November to 4 December 2009 (IEEE Computer Society Press, Los Alamitos, 2009), pp. 1-6

3. JJ van de Beek, O Edfors, M Sandell, SK Wilson, PO Börjesson, On channel estimation in OFDM systems, in IEEE Conference on Vehicular Technology, Chicago, 25-28 July 1995, vol. 2 (Society Staff IEEE, Los Alamitos, 1995), pp. 815-819

4. TA Summers, SG Wilson, SNR mismatch and online estimation in turbo decoding. IEEE Trans. Commun. 46, 421-423 (1998)

5. DR Pauluzzi, A comparison of SNR estimation techniques for the AWGN channel. IEEE Trans. Commun. 48(10), 1681-1691 (2000)

6. G Ren, Y Chang, $\mathrm{H}$ Zhang, A new SNR's estimator for QPSK modulations in an AWGN channel. IEEE Trans. Circuits Syst. 52(6), 336-338 (2005)

7. B Li, R Di Fazio, A Zeira, A low bias algorithm to estimate negative SNRs in an AWGN channel. IEEE Commun. Lett. 6(11), 469-471 (2002)

8. TR Benedict, TT Soong, The joint estimation of signal and noise from the sum envelope. IEEE Trans. Inf. Theory. 13, 447-454 (1967)

9. S Kay, Chapter 12, in Fundamentals of Statistical Signal Processing: Estimation Theory (Prentice-Hall PTR, Upper Saddle River, 2003), pp. 388-391

10. HXu, G Wei, J Zhu, A novel SNR estimation algorithm for OFDM, in IEEE Vehicular Technology Conference vol. 5 (IEEE, Los Alamitos, 2005), pp. 3068-3071

11. S Boumard, Novel noise variance and SNR estimation algorithm for wireless MIMO OFDM systems, in IEEE Global Telecommunications Conference, vol. 3 (IEEE Computer Society Press, Los Alamitos, 2003), pp. 1330-1334

12. G Ren, H Zhang, Y Chang, SNR estimation algorithm based on the preamble for OFDM systems in frequency selective channels. IEEE Trans. Commun. 57(8), 2230-2234 (2009)

13. $X X u, Y$ Jing, $X Y u$, Subspace-based noise variance and SNR estimation for OFDM systems, in IEEE Mobile Radio Applications Wireless Communication Networking Conference vol. 1 (IEEE Computer Society Press, Los Alamitos, 2005), pp. 23-26

14. M Biguesh, AB Gershman, Training-based MIMO channel estimation: a study of estimator tradeoffs and optimal training signals. IEEE Trans. Signal Proc. 54(3), 884-893 (2006)

15. S Coleri, M Ergen, A Puri, A Bahai, A study of channel estimation in OFDM systems, in Proceedings of IEEE 56th Vehicular Technology Conference (VTC 2002-Fall 2002), vol. 2 (IEEE Computer Society Press, Los Alamitos, 2002), pp. 894-898

16. O Edfords, M Sandell, JJ van de Beek, SK Wilson, PO Börjesson, OFDM channel estimation by singular value decomposition. IEEE Trans. Commun. 46(7), 931-939 (1998)

17. V Savaux, A Skrzypczak, Y Louët, M Djoko-Kouam, Near LMMSE channel estimation performance with artificial channel at receiver for OFDM systems, in SPAWC 2012, (Cesme, 17-20 June 2012)

18. S Haykin, Adaptive Filter Theory (Prentice Hall, Englewood Cliffs, 2001)
19. Y Kojima, H Tomeba, K Takeda, F Adachi, RLS channel estimation with adaptive forgetting factor for DS-CDMA frequency-domain equalization. IEICE Trans, Commun. E92-B(5), 1457-1465 (2009)

20. D Schafhuber, G Matz, F Hlawatsch, Adaptive Wiener filters for timevarying channel estimation in wireless OFDM systems, in ICASSP'03, vol. 4, Hong-Kong, 6-10 April 2003 (IEEE Press, Piscataway, 2003), pp. 688-691

21. G Matz, Recursive MMSE estimation of wireless channels based onTraining data and structured correlation learning, in SSP'05, Bordeaux, 17-20 July 2005 (IEEE Press, Piscataway, 2005), pp. 1342-1347

22. H Hijazi, L Ros, Joint data QR-detection and Kalman estimation for OFDM time-varying rayleigh channel complex gains. IEEE Trans. Commun. 58, 170-178 (2010)

23. S Buzzi, M Lops, S Sardellitti, Performance of iterative data detection and channel estimation for single-antenna and multiple-antennas wireless communications. IEEE Trans. Vehicular Tech. 53(4), 1085-1104 (2004)

24. E Panayirci, H Senol, HV Poor, Joint channel estimation, equalization, and data detection for OFDM systems in the presence of very high mobility. IEEE Trans. Commun. 58(8), 4225-4238 (2010)

25. T Zemen, CF Mecklenbräuker, J Wehinger, RR Müller, Iterative joint time-variant channel estimation and multi-user detection for MC-CDMA IEEE Trans. Wireless Commun. 5(6), 1469-1478 (2006)

26. RC de Lamare, R Sampaio-Neto, A Hjorungnes, Joint iterative interference cancellation and parameter estimation for CDMA systems. IEEE Commun. Lett. 11(12), 916-918 (2007)

27. AP Dempster, NM Laird, DB Rubin, Maximum likelihood from incomplete data via the EM algorithm. J. R. Stat. Soc. Ser. B. 39, 1-38 (1977)

28. X Wautelet, C Herzet, A Dejonghe, J Louveaux, L Vandendorpe, Comparison of EM-based algorithms for MIMO channel estimation. IEEE Trans. Commun. 55, 216-226 (2007)

29. T Krisjansson, B Frey, L Deng, A Acero, Joint estimation of noise and channel distortion in a generalized EM framework, in IEEE Workshop on ASRU'01 (IEEE Press, Piscataway, 2001), pp. 155-158

30. Z Baranski, A Haimovich, J Garcia-Frias, EM-based iterative receiver for space-time coded modulation with noise variance estimation, in GLOBECOM'02, vol. 1 (IEEE Press, Piscataway, 2002), pp. 355-359

31. SH Wu, U Mitra, CCJ Kuo, Iterative joint channel estimation and multiuser detection for DS-CDMA in frequency-selective fading channels. IEEE Trans. Signal Proc. 56(7), 3261-3277 (2008)

32. O Rousseaux, G Leus, P Stoica, M Moonen, Gaussian maximum-likelihood channel estimation with short training sequences. IEEE Trans. Wireless Commun. 4(6) (2005)

33. A Wiesel, YC Eldar, A Beck, Maximum likelihood estimation in linear models with a gaussian model matrix. IEEE Signal Proc. Lett. 13(5), 292-295 (2006)

34. V Savaux, Y Louët, M Djoko-Kouam, A Skrzypczak, An iterative and joint estimation of SNR and frequency selective channel for OFDM systems, in European Wireless, (Poznan, Poland, 1-7 April 2012)

35. ETSI: Digital Radio Mondiale (DRM): system specification. Technical Report ETSI ES 201980 V 3.1.1, ETSI (2009)

36. ETSI: Digital Video Broadcasting (DVB) framing structure, channel coding and modulation for digital terrestrial television. Technical Report ETSI EN 300744 V1.5.1, ETSI (2004)

doi:10.1186/1687-6180-2013-128

Cite this article as: Savaux et al:: Application of a joint and iterative MMSE-based estimation of SNR and frequency-selective channel for OFDM systems. EURASIP Journal on Advances in Signal Processing 2013 2013:128. 